\title{
Effects of partial coherence on correlation functions measured by $x$-ray photon correlation spectroscopy
}

\author{
C. Gutt, ${ }^{1}$ T. Ghaderi, ${ }^{2,3}$ M. Tolan, ${ }^{2}$ S. K. Sinha,${ }^{3}$ and G. Grübel ${ }^{1}$ \\ ${ }^{1}$ HASYLAB at DESY, Notkestrasse 85, Hamburg, Germany \\ ${ }^{2}$ Experimentelle Physik I, University Dortmund, Dortmund, Germany \\ ${ }^{3}$ Physics Department, University of California, San Diego, La Jolla, California 92037, USA \\ (Received 11 October 2007; revised manuscript received 28 January 2008; published 26 March 2008)
}

\begin{abstract}
We present a rigorous description of the effects of partial coherence and detector resolution on intensity autocorrelation functions as they can be measured by X-ray photon correlation spectroscopy (XPCS). Based on the Huygens-Fresnel propagation law and on the first Born approximation, we derive a general expression for the normalized intensity autocorrelation function. We calculate how the mutual coherence function of the $\mathrm{x}$-ray beam propagates from an aperture to the sample and how it propagates after the scattering process to the detector area and consequently influences the intensity autocorrelation function. We illustrate our calculation with examples of XPCS intensity autocorrelation functions of liquid surfaces calculated for grazing incidence geometry.
\end{abstract}

DOI: 10.1103/PhysRevB.77.094133

PACS number(s): 61.05.cc, 68.03.Kn

\section{INTRODUCTION}

In the last few years the technique of $\mathrm{x}$-ray photon correlation spectroscopy (XPCS) has increasingly been used to study dynamic properties of liquid or soft matter surfaces. ${ }^{1-11}$ At third-generation synchrotrons it is nowadays possible to produce intense $\mathrm{x}$-ray beam with a high degree of coherence. The scattered intensity distribution of such an x-ray beam coming from a rough surface provides a speckle pattern due to destructive and constructive interference. ${ }^{12,13}$ At liquid or soft matter surfaces the roughness is generated by thermally excited capillary waves, and the related speckle distribution changes on the same time scale as the surface fluctuation. The measurements of the temporal intensity autocorrelation function of these speckles contain information about the dynamic properties of these surfaces. For highly viscous liquids the thermally excited waves are overdamped. The corresponding autocorrelation function shows a time-dependent exponential decay. The situation is different for liquids with low viscosity. The capillary waves propagate and thus the autocorrelation function shows an oscillatory behavior., Previous experiments with optical photon correlation spectroscopy techniques, known as dynamical light scattering (DLS), have been used to investigate various fluid systems. ${ }^{14,15}$ However for DLS measurements of lowviscosity liquids the experimentally determined damping constants (viscosities) were greater than those obtained by conventional techniques. This issue was first addressed and explained by Langevin. ${ }^{14,16}$ The effect was related to broadening (resolution) effects of the experimental setup. Similar discrepancies between observed and theoretically expected damping constants have been observed studying the surface fluctuations of liquid water in an experiment using surfacesensitive XPCS. ${ }^{5}$

Most treatments of the effects of beam divergence, energy spread, etc. use the resolution function folding procedure in which the observed intensities are calculated in terms of a convolution of the actual scattering function $S(q)$ with an instrumental resolution function $\widetilde{R}(q)$ (see, e.g., Ref. 17). The
Fourier transform of $\widetilde{R}(q)$ is related to the coherence volume, so that coherence length and resolution widths are regarded as conjugate quantities. In a rigorous treatment it has been shown that this approach is valid only in the case of a completely incoherent source and in the Fraunhofer limit. ${ }^{18}$ These two conditions are seldom met in experiments applying x-ray photon correlation spectroscopy. However, a detailed analysis shows that under certain circumstances, such as, e.g., a spatially homogeneous sample, a similar resolution folding approach can be applied to XPCS experiments. ${ }^{19}$

The measured quantity in XPCS experiments is the timedependent intensity autocorrelation function. In case of Gaussian fluctuations the intensity correlation function can be connected with the intermediate scattering function of the system, $f(q, \tau)$, via the Siegert relation, and one obtains

$$
\frac{\langle I(q, t) I(q, t+\tau)\rangle}{\langle I(q)\rangle^{2}}=1+|f(q, \tau)|^{2} .
$$

Following a paper by Pusey, ${ }^{20}$ the effects of partial coherence are usually accounted for by introducing a contrast factor $\beta$ in Eq. (1),

$$
\frac{\langle I(q, t) I(q, t+\tau)\rangle}{\langle I(q)\rangle^{2}}=1+\beta^{2}|f(q, \tau)|^{2},
$$

leading to a convenient separation of beam properties and sample properties. The conditions necessary for this separation are that the coherence length of the light is larger than the correlation length of the sample and that the scattering volume is also much larger than the correlation length of the sample fluctuations. It turns out that these conditions are usually not satisfied for $\mathrm{x}$ rays, and especially not under grazing incidence conditions.

In this paper, we will therefore derive a rigorous theoretical description of the effects of partial coherence and detector resolution on intensity autocorrelation functions. Based on the Huygens-Fresnel propagation law and on the first Born approximation, we derive a general expression for the normalized intensity autocorrelation function. We calculate 


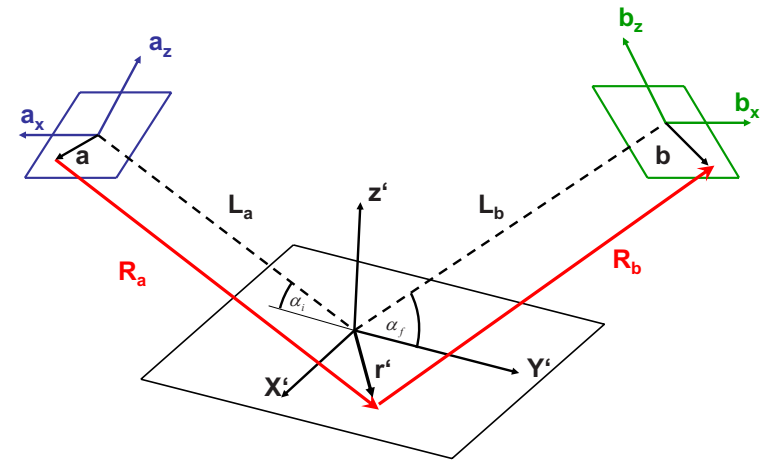

FIG. 1. (Color online) Schematic view of the scattering geometry. The beam emerges from the aperture A. The line $L_{a}$ is parallel to the mean beam direction and connects the center of the aperture and the center of the sample. $L_{b}$ is parallel to the mean outgoing beam direction and connects the center of the sample with the center of the detector aperture B. An x-ray beam emerges from A at position a, propagates along the line $R_{a}$, falls onto the sample at position $\mathbf{r}$, and is then scattered along the line $R_{b}$ into the detector at position $\mathbf{b}$.

how the mutual coherence function (MCF) of the x-ray beam propagates from an aperture to the sample, and how it propagates after the scattering process to the detector area and consequently influences the intensity autocorrelation function. We illustrate this with examples of XPCS intensity autocorrelation functions of liquid surfaces calculated for grazing incidence geometry. We show that coherence effects enter in a more complicated way than speckle visibility, as they affect the functional form of the correlation function also. With this we are able to explain prominent features of coherence effects in surface XPCS experiments such as (i) the observation of heterodyne mixing, i.e., the occurrence of both first- and second-order correlation functions in the intensity autocorrelation function, and (ii) larger effective damping constants due to the smearing with a resolution function.

We proceed in the next section to evaluate the intensity autocorrelation functions based on the Huygens-Fresnel theory. We will refer to the notation and terminology as used in Ref. 18.

\section{INTENSITY CORRELATION FUNCTIONS FROM HUYGENS-FRESNEL THEORY}

Figure 1 displays a schematic view of the setup considered. The beam emerges from an aperture A, the plane of which is normal to the mean direction of the beam and the line joining its center to the sample center at a distance $L_{a}$. The angle $\alpha_{i}$ denotes the angle between this line and the sample surface. The beam is then transmitted through an aperture B oriented normally to the average direction of the scattered beam at a distance $L_{b}$ from the center of the sample. Behind this aperture a detector is placed. The angle $\alpha_{f}$ denotes the angle between this line and the sample surface. The vectors $\mathbf{a}$ and $\mathbf{b}$ represent the coordinates within the aperture $\mathrm{A}$ and detector B, respectively.
The observed quantity in XPCS experiments is the correlation of the X-ray intensity $I\left(\mathbf{r}_{1}, t_{1}\right)$ measured at time $t_{1}$ at position $\mathbf{r}_{1}$ with the intensity $I\left(\mathbf{r}_{2}, t_{t}\right)$ measured at a later time $t_{2}$ and at a different position $\mathbf{r}_{2}$,

$$
G_{2}\left(\mathbf{r}_{1}, \mathbf{r}_{2}, t_{2}-t_{1}\right)=\left\langle I\left(\mathbf{r}_{1}, t_{1}\right) I\left(\mathbf{r}_{2}, t_{2}\right)\right\rangle,
$$

integrated over all possible positions $\mathbf{r}_{1}$ and $\mathbf{r}_{2}$ within the finite detector area. Alternatively, one can express the intensity correlation function $G_{2}\left(\mathbf{r}_{1}, \mathbf{r}_{2}, t_{2}-t_{1}\right)$ with the aid of the scattered fields as

$$
G_{2}\left(\mathbf{r}_{1}, \mathbf{r}_{2}, t_{2}-t_{1}\right)=\left\langle U_{s}^{*}\left(\mathbf{r}_{1}, t_{1}\right) U_{s}\left(\mathbf{r}_{1}, t_{1}\right) U_{s}^{*}\left(\mathbf{r}_{2}, t_{2}\right) U_{s}\left(\mathbf{r}_{2}, t_{2}\right)\right\rangle,
$$

in which $U_{s}(\mathbf{r}, t)$ is a complex scalar representation of the scattered electric field. The rigorous evaluation of Eq. (4) with the field expressions for the scattered radiation is in general a nontrivial task. One way to approach a solution for the intensity autocorrelation function is based on the statistical properties of the scattered field $U_{s}(r, t)$. If the scattered fields are of zero mean, obey Gaussian statistics, and depend on time differences only, the Gaussian momentum theorem can be applied, yielding finally a modified version of the well-known Siegert relation. It is, however, not always guaranteed that the scattered fields have the properties required for the momentum theorem. In order to proceed, we assume the fields at the detector to be approximately Gaussian so we can in the following apply the momentum theorem and obtain

$$
G_{2}\left(\mathbf{r}_{1}, \mathbf{r}_{2}, \tau\right)=\left\langle I\left(\mathbf{r}_{1}, 0\right)\right\rangle\left\langle I\left(\mathbf{r}_{1}, \tau\right)\right\rangle+\left|\left\langle U_{s}^{*}\left(\mathbf{r}_{1}, 0\right) U_{s}\left(\mathbf{r}_{2}, \tau\right)\right\rangle\right|^{2},
$$

with $\tau=t_{2}-t_{1}$. In the limit of point detection and stationary fields, i.e., $\mathbf{r}_{1} \approx \mathbf{r}_{2}$, the above relation reduces to the widely used Siegert relation $G_{2}(\mathbf{r}, \tau)=\langle I(\mathbf{r}, 0)\rangle^{2}$ $+\left|\left\langle U_{s}^{*}(\mathbf{r}, 0) U_{s}(\mathbf{r}, \tau)\right\rangle\right|^{2}$.

In order to proceed one has to determine the mutual coherence function of the scattered fields $\Gamma_{s}\left(\mathbf{r}_{1}, \mathbf{r}_{2}, \tau\right)$ $=\left\langle U_{s}^{*}\left(\mathbf{r}_{1}, 0\right) U_{s}\left(\mathbf{r}_{2}, \tau\right)\right\rangle$. In the first Born approximation the MCF of the scattered fields depends on both the electron density correlations $C_{\rho \rho}\left(\mathbf{r}_{1}^{\prime}, \mathbf{r}_{2}^{\prime}, \tau\right)=\left\langle\rho\left(\mathbf{r}_{1}^{\prime}, 0\right) \rho\left(\mathbf{r}_{2}^{\prime}, \tau\right)\right\rangle$ of the sample and the mutual coherence function of the incident fields $J_{i}\left(\mathbf{r}_{1}^{\prime}, \mathbf{r}_{2}^{\prime}\right)$ according to

$$
\begin{aligned}
\Gamma_{s}\left(\mathbf{L}_{b}+\mathbf{b}_{1}, \mathbf{L}_{b}+\mathbf{b}_{2}, \tau\right)= & r_{e}^{2} \iint_{V^{\prime}} d \mathbf{r}_{1}^{\prime} d \mathbf{r}_{2}^{\prime} C_{\rho \rho}\left(\mathbf{r}_{1}^{\prime}, \mathbf{r}_{2}^{\prime}, \tau\right) \\
& \times \frac{J_{i}\left(\mathbf{r}_{1}^{\prime}, \mathbf{r}_{2}^{\prime}\right)}{R_{b 1} R_{b 2}} e^{i \omega_{0}\left(\tau-\left(R_{b 2}-R_{b 1}\right) / c\right),}
\end{aligned}
$$

where $\mathbf{r}^{\prime}$ denotes the coordinates within the sample and $r e$ is the classical electron radius. The integral extends over the volume of the sample and $R_{b 1}=\left|\mathbf{L}_{b}+\mathbf{b}_{1}-\mathbf{r}_{1}^{\prime}\right|, R_{b 2}=\mid \mathbf{L}_{b}+\mathbf{b}_{2}$ $-\mathbf{r}_{2}^{\prime} \mid$ are the distances from the sample to the points of detection, where $\mathbf{L}_{b}$ is specified by the mean direction of the scattered beam and the distance to the detector. In order to arrive at Eq. (6), the implicit assumption has been made that the time difference for two different rays to reach the detector at $\mathbf{b}_{1}$ and $\mathbf{b}_{2}$ from points $\mathbf{r}_{1}^{\prime}$ and $\mathbf{r}_{2}^{\prime}$, respectively, is negligible compared to the times $\tau$ involved in the correlation 
function. This may not be true if one wishes to study femtosecond phenomena using $\mathrm{x}$-ray free electron laser (XFEL) sources. But our formulas can easily be generalized to take that into account.

The MCF of the incident fields is itself the result of the fields propagating from a finite aperture A toward the sample. The field correlations between two points $\mathbf{a}_{1}$ and $\mathbf{a}_{2}$ on the planar aperture area A may be described by the mutual coherence function $J_{a}\left(\mathbf{a}_{1}, \mathbf{a}_{2}\right)$. The relation between the mutual coherence functions at the aperture and the sample position can be deduced from the Cittert-Zernike propagation law

$$
J_{i}\left(\mathbf{r}_{1}^{\prime}, \mathbf{r}_{2}^{\prime}\right)=\iint_{A} d \mathbf{a}_{1} d \mathbf{a}_{2} J_{a}\left(\mathbf{a}_{1}, \mathbf{a}_{2}\right) \frac{e^{i k_{0}\left(R_{a 2}-R_{a 1}\right)}}{R_{a 1} R_{a 2}} \Lambda_{1}^{*} \Lambda_{2}
$$

with the inclination factors $\Lambda_{1,2}=i \frac{k_{0}}{2 \pi} \cos \theta_{1,2}$, where $\theta$ represents the angle between $R_{a}$ and the normal vector of the aperture plane and $R_{a 1}=\left|\mathbf{L}_{a}-\mathbf{a}_{1}+\mathbf{r}_{1}^{\prime}\right|, \quad R_{a 2}=\left|\mathbf{L}_{a}-\mathbf{a}_{2}+\mathbf{r}_{2}^{\prime}\right|$, where $\mathbf{L}_{a}$ is specified by the distance $L_{a}$ and the mean direction of the incident beam. In our case the incident aperture planes and the detector aperture planes are normal to the average incident and scattered beam from the samples. That is, we have $\theta \ll 1$, which means we can write $\Lambda_{1,2} \approx i \frac{k_{0}}{2 \pi}$.

Let us replace the path length differences in the denominators of Eqs. (6) and (7) with their mean distances $L_{a}$ and $L_{b}$, respectively. As the detector is fixed in space we also drop the formal $\mathbf{L}_{b}$ dependence, and we obtain

$$
\begin{aligned}
\Gamma_{s}\left(\mathbf{b}_{1}, \mathbf{b}_{2}, \tau\right)= & \left(\frac{r_{e}}{L_{a} L_{b} \lambda}\right)^{2} e^{i \omega_{0} \tau} \iint_{V^{\prime}} d \mathbf{r}_{1}^{\prime} d \mathbf{r}_{2}^{\prime} C_{\rho \rho}\left(\mathbf{r}_{1}^{\prime}, \mathbf{r}_{2}^{\prime}, \tau\right) \\
& \times \iint_{A} d \mathbf{a}_{1} d \mathbf{a}_{2} J_{a}\left(\mathbf{a}_{1}, \mathbf{a}_{2}\right) e^{i k_{0} \Delta l}
\end{aligned}
$$

with $\Delta l=\left(R_{a 2}-R_{a 1}\right)+\left(R_{b 2}-R_{b 1}\right)$ being the total path difference between two $\mathrm{x}$ rays that are propagating from a finite slit area A via a scattering process toward different points on the detection screen $\mathrm{B}$.

Expanding $\Delta l$ up to second order (see, e.g., Ref. 18), we introduce a resolution function $R_{1}\left(\mathbf{r}_{1}^{\prime}, \mathbf{r}_{2}^{\prime}, \mathbf{b}_{1}, \mathbf{b}_{2}\right)$, which is

$$
\begin{aligned}
& R_{1}\left(\mathbf{r}_{1}^{\prime}, \mathbf{r}_{2}^{\prime}, \mathbf{b}_{1}, \mathbf{b}_{2}\right) \\
& =e^{i \kappa_{a}\left[\mathbf{r}_{2}^{\prime 2}-\left(\mathbf{r}_{2}^{\prime} \cdot \hat{\mathbf{k}}_{i}\right)^{2}-\mathbf{r}_{1}^{\prime 2}+\left(\mathbf{r}_{1} \cdot \hat{\mathbf{k}}_{i}\right)^{2}\right]} \\
& \quad \times \iint d \mathbf{a}_{1} d \mathbf{a}_{2} J_{a}\left(\mathbf{a}_{1}, \mathbf{a}_{2}\right) e^{i \kappa_{a}\left[\mathbf{a}_{2}^{2}-\mathbf{a}_{1}^{2}-2\left(\mathbf{a}_{2} \cdot \mathbf{r}_{2}^{\prime}-\mathbf{a}_{1} \cdot \mathbf{r}_{1}^{\prime}\right)\right]} \\
& \quad \times e^{i \kappa_{b}\left(b_{2}^{2}-b_{1}^{2}\right)} e^{-i 2 \kappa_{b}\left(\mathbf{b}_{2} \cdot \mathbf{r}_{2}^{\prime}-\mathbf{b}_{1} \cdot \mathbf{r}_{1}^{\prime}\right)} e^{i \kappa_{b}\left[\mathbf{r}_{2}^{\prime 2}-\left(\mathbf{r}_{2}^{\prime} \cdot \hat{\mathbf{k}}_{f}\right)^{2}-\mathbf{r}_{1}^{\prime 2}+\left(\mathbf{r}_{1}^{\prime} \cdot \hat{\mathbf{k}}_{f}\right)^{2}\right]}
\end{aligned}
$$

with $\kappa_{a}=k_{0} / 2 L_{a}$ and $\kappa_{b}=k_{0} / 2 L_{b}$. The nominal wave vector transfer is $\mathbf{q}=k_{0}\left(\hat{\mathbf{k}}_{f}-\hat{\mathbf{k}}_{i}\right)$ with the mean directions of the incident beam $\hat{\mathbf{k}}_{i}$ and the scattered beam $\hat{\mathbf{k}}_{f} . \mathbf{k}_{0}=2 \pi / \lambda$ denotes the wave number.

Up to now we have implicitly assumed a completely monochromatic beam. Nonmonochromaticity of the beam shortens the longitudinal coherence length. The longitudinal coherence length can be included in our formalism by introducing a factor

$$
W_{1}\left(\mathbf{r}_{1}^{\prime}, \mathbf{r}_{2}^{\prime}\right)=\int d(\Delta \omega) F(\Delta \omega) \exp ^{-i \Delta \omega / \bar{\omega} \mathbf{q}\left(\mathbf{r}_{1}^{\prime}-\mathbf{r}_{2}^{\prime}\right)}
$$

into the expression Eq. (9) for the resolution function. $F(\Delta \omega)$ is the distribution of frequencies of the incident beam about the average frequency $\bar{\omega}$. With this, we obtain an expression for the general resolution function,

$$
R_{G}\left(\mathbf{r}_{1}^{\prime}, \mathbf{r}_{2}^{\prime}, \mathbf{b}_{1}, \mathbf{b}_{2}\right)=R_{1}\left(\mathbf{r}_{1}^{\prime}, \mathbf{r}_{2}^{\prime}, \mathbf{b}_{1}, \mathbf{b}_{2}\right) W_{1}\left(\mathbf{r}_{1}^{\prime}, \mathbf{r}_{2}^{\prime}\right) .
$$

This allows us to obtain a compact notation for $\Gamma_{s}\left(\mathbf{q}, \mathbf{b}_{1}, \mathbf{b}_{2}, \tau\right)$,

$$
\begin{aligned}
\Gamma_{s}\left(\mathbf{q}, \mathbf{b}_{1}, \mathbf{b}_{2}, \tau\right)= & \left(\frac{r_{e}}{L_{a} L_{b} \lambda}\right)^{2} \iint_{V^{\prime}} d \mathbf{r}_{1}^{\prime} d \mathbf{r}_{2}^{\prime} C_{\rho \rho}\left(\mathbf{r}_{1}^{\prime}, \mathbf{r}_{2}^{\prime}, \tau\right) \\
& \times R_{G}\left(\mathbf{r}_{1}^{\prime}, \mathbf{r}_{2}^{\prime}, \mathbf{b}_{1}, \mathbf{b}_{2}\right) e^{-i \mathbf{q}\left(\mathbf{r}_{2}^{\prime}-\mathbf{r}_{1}^{\prime}\right)}
\end{aligned}
$$

where the phase factor $e^{i \omega_{0} \tau}$ has been omitted, because it drops out in the Siegert relation. If the density correlations are spatially homogeneous, we can replace $C_{\rho \rho}\left(\mathbf{r}_{1}^{\prime}, \mathbf{r}_{2}^{\prime}, \tau\right)$ by $C_{\rho \rho}\left(\mathbf{r}_{1}^{\prime}-\mathbf{r}_{2}^{\prime}, \tau\right)$ and obtain, with

$$
R_{2}\left(\mathbf{R}, \mathbf{b}_{1}, \mathbf{b}_{2}\right)=\int d \mathbf{r}_{1}^{\prime} R_{G}\left(\mathbf{r}_{1}^{\prime}, \mathbf{r}_{1}^{\prime}+\mathbf{R}, \mathbf{b}_{1}, \mathbf{b}_{2}\right)
$$

where $R=\left|\mathbf{r}_{2}^{\prime}-\mathbf{r}_{1}^{\prime}\right|$, an expression for the correlation function,

$$
\Gamma_{s}\left(\mathbf{q}, \mathbf{b}_{1}, \mathbf{b}_{2}, \tau\right)=\left(\frac{r_{e}}{L_{a} L_{b} \lambda}\right)^{2} \int d \mathbf{R} C_{\rho \rho}(\mathbf{R}, \tau) R_{2}\left(\mathbf{R}, \mathbf{b}_{1}, \mathbf{b}_{2}\right) e^{-i \mathbf{q} \mathbf{R}},
$$

which then yields with the convolution theorem the familiar convolution in reciprocal space

$$
\Gamma_{s}\left(\mathbf{q}, \mathbf{b}_{1}, \mathbf{b}_{2}, \tau\right)=\left(\frac{r_{e}}{L_{a} L_{b} \lambda}\right)^{2} \int d \mathbf{q}^{\prime} \tilde{C}_{\rho \rho}\left(\mathbf{q}^{\prime}, \tau\right) \widetilde{R}_{2}\left(\mathbf{q}-\mathbf{q}^{\prime}, \mathbf{b}_{1}, \mathbf{b}_{2}\right),
$$

where the tilde denotes the Fourier transform of each function.

To obtain finally the intensity autocorrelation function $G_{2}(\mathbf{q}, \tau)$ we have to integrate over the detector area $B$, and we find

$$
\begin{aligned}
G_{2}(\mathbf{q}, \tau)= & \iint_{B} d \mathbf{b}_{1} d \mathbf{b}_{2}\left\langle\Gamma_{s}\left(\mathbf{q}, \mathbf{b}_{1}, \mathbf{b}_{1}, 0\right)\right\rangle\left\langle\Gamma_{s}\left(\mathbf{q}, \mathbf{b}_{2}, \mathbf{b}_{2}, 0\right)\right\rangle \\
& +\iint_{B} d \mathbf{b}_{1} d \mathbf{b}_{2}\left|\Gamma_{s}\left(\mathbf{q}, \mathbf{b}_{2}, \mathbf{b}_{1}, \tau\right)\right|^{2}
\end{aligned}
$$

We will first concentrate on the time-dependent part in the intensity autocorrelation function and write 


$$
\begin{aligned}
\int d & \mathbf{b}_{1} d \mathbf{b}_{2}\left|\Gamma_{s}\left(\mathbf{q}, \mathbf{b}_{1}, \mathbf{b}_{2}, \tau\right)\right|^{2} \\
= & \left(\frac{r_{e}}{L_{a} L_{b} \lambda}\right)^{2} \int d \mathbf{q}^{\prime} d \mathbf{q}^{\prime \prime} \widetilde{C}_{\rho \rho}\left(q^{\prime}, \tau\right) \widetilde{C}_{\rho \rho}\left(q^{\prime \prime}, \tau\right) \\
& \times \int d \mathbf{b}_{1} d \mathbf{b}_{2} e^{-\left(\mathbf{b}_{1}^{2}+\mathbf{b}_{2}^{2}\right) / \beta^{2}} \widetilde{R}_{2}\left(\mathbf{q}-\mathbf{q}^{\prime}, \mathbf{b}_{1}, \mathbf{b}_{2}\right) \\
& \times \widetilde{R}_{2}\left(\mathbf{q}-\mathbf{q}^{\prime \prime}, \mathbf{b}_{1}, \mathbf{b}_{2}\right),
\end{aligned}
$$

where we introduced a Gaussian pupil function for the detector area characterized by the detector opening $\beta$. In order to proceed, explicit representations of the general resolution function $R_{2}\left(\mathbf{R}, \mathbf{b}_{1}, \mathbf{b}_{2}\right)$ are needed. We consider again a monochromatic beam with unlimited longitudinal coherence length which is valid for small $\mathbf{q}$ values or if $\mathbf{q}$ is perpendicular to $\left(\mathbf{r}_{1}^{\prime}-\mathbf{r}_{2}^{\prime}\right)$. A popular approach is then to consider a Gaussian Schell-model source in which the intensity distribution and the mutual correlation functions are modeled by Gaussian functions; this also has the advantage that analytical solutions of many integrals exists. By using such a model source we obtain for the resolution function

$$
\begin{aligned}
\widetilde{R}_{2}\left(\mathbf{q}, \mathbf{b}_{1}, \mathbf{b}_{2}\right)= & \pi \Delta \exp \left\{-\left(\mathbf{b}_{1}-\mathbf{b}_{2}\right)^{2} \Delta^{2} \kappa_{B}^{2}-\Delta^{2}\left[\mathbf{q}-\left(\mathbf{b}_{1}\right.\right.\right. \\
& \left.\left.\left.+\mathbf{b}_{2}\right) \kappa_{B}\right]^{2}\right\}
\end{aligned}
$$

with the parameter $\Delta$, which comprises parameters like slit opening, transverse coherence lengths, etc. Explicit expressions of $\Delta$ for different model sources have been calculated in Ref. 19. Performing the integration of the resolution functions $\widetilde{R}_{2}$ over the detector area $B$ yields an expression for the resolution function:

$$
\begin{aligned}
F\left(\mathbf{q}, \mathbf{q}^{\prime}, \mathbf{q}^{\prime \prime}\right)= & \frac{\pi^{2} \Delta^{2}}{1 / \beta^{2}+4 \Delta^{2} \kappa_{B}^{2}} \exp \left(-\Delta^{2}\left(\mathbf{q}-\mathbf{q}^{\prime}\right)^{2}-\Delta^{2}\left(\mathbf{q}-\mathbf{q}^{\prime \prime}\right)^{2}\right. \\
& \left.-\left(2 \mathbf{q}-\mathbf{q}^{\prime}-\mathbf{q}^{\prime \prime}\right)^{2} \frac{\kappa_{B}^{2} \beta^{2} \Delta^{4}}{1+4 \beta^{2} \Delta^{2} \kappa_{B}^{2}}\right)
\end{aligned}
$$

so we can write

$$
\begin{aligned}
& \int d \mathbf{b}_{1} d \mathbf{b}_{2}\left|\Gamma_{s}\left(\mathbf{q}, \mathbf{b}_{1}, \mathbf{b}_{2}, \tau\right)\right|^{2} \\
& \quad=\int d \mathbf{q}^{\prime} d \mathbf{q}^{\prime \prime} \widetilde{C}_{\rho \rho}\left(\mathbf{q}^{\prime}, \tau\right) \widetilde{C}_{\rho \rho}\left(\mathbf{q}^{\prime \prime}, \tau\right) F\left(\mathbf{q}, \mathbf{q}^{\prime}, \mathbf{q}^{\prime \prime}\right) .
\end{aligned}
$$

Similarly, we can express the detected intensity as an integral over the detector area and write

$$
\begin{aligned}
\langle I(\mathbf{q})\rangle & =\int d \mathbf{b}_{1} \Gamma_{s}\left(\mathbf{q}, \mathbf{b}_{1}, \mathbf{b}_{1}\right) \\
& =\int d \mathbf{q}^{\prime} \widetilde{C}_{\rho \rho}\left(q^{\prime}, 0\right) \int d \mathbf{b}_{1} e^{-\mathbf{b}_{1}^{2} / \beta^{2}} \widetilde{R}_{2}\left(\mathbf{q}-\mathbf{q}^{\prime}, \mathbf{b}_{1}, \mathbf{b}_{1}\right) \\
& =\int d \mathbf{q}^{\prime} \widetilde{C}_{\rho \rho}\left(q^{\prime}, 0\right) H\left(\mathbf{q}, \mathbf{q}^{\prime}\right)
\end{aligned}
$$

with the resolution function

$$
H\left(\mathbf{q}, \mathbf{q}^{\prime}\right)=\sqrt{\frac{\pi^{2} \Delta^{2}}{1 / \beta^{2}+4 \Delta^{2} \kappa_{B}^{2}}} \exp \left(-\frac{\left(\mathbf{q}-\mathbf{q}^{\prime}\right)^{2} \Delta^{2}}{1+4 \beta^{2} \Delta^{2} \kappa_{B}^{2}}\right) .
$$

Finally we arrive at an expression for the normalized intensity autocorrelation function,

$$
g_{2}(\mathbf{q}, \tau)=1+\frac{\iint d \mathbf{q}^{\prime} d \mathbf{q}^{\prime \prime} \tilde{C}_{\rho \rho}\left(\mathbf{q}^{\prime}, \tau\right) \tilde{C}_{\rho \rho}\left(\mathbf{q}^{\prime \prime}, \tau\right) F\left(\mathbf{q}, \mathbf{q}^{\prime}, \mathbf{q}^{\prime \prime}\right)}{\left(\int d \mathbf{q}^{\prime} \tilde{C}_{\rho \rho}\left(q^{\prime}, 0\right) H\left(\mathbf{q}, \mathbf{q}^{\prime}\right)\right)^{2}} .
$$

This expression is the main result of our paper. It should be clearly noted that expression (23) is substantially different from the simplified incoherent approaches, where all effects of partial coherence are condensed into a single prefactor expressing the speckle contrast.

An important parameter quantifying the effects of partial coherence is the ratio $\Delta q / q$ between wave vector spread and wave vector value. If this value is small, effects of partial coherence can usually be neglected, while the influence of the opening of the detector still persists. If this ratio is large, as, for example, for the surface scattering geometry, the effects of partial coherence are very important, as is illustrated in the following examples.

\section{A. Surface scattering geometry}

The electron density correlation function for liquid surfaces can be approximated by a convenient model for the electron density at a liquid interface. The density consists of a mean equilibrium value $\bar{\rho}$ plus a fluctuating part $\Delta \rho$ representing the surface height fluctuations around a mean surface. Then the volume integral in Eq. (23) can be converted into a surface integral with the density-density correlation function replaced by the usual expression ${ }^{21}$

$$
\frac{\bar{\rho}^{2}}{q_{z}^{2}} e^{-q_{z}^{2} \sigma^{2}}\left[\delta\left(q_{\|}\right)+q_{z}^{2} \widetilde{C}_{z z}\left(q_{\|}, \tau\right)\right]
$$

containing the time-dependent height-height correlation function $\widetilde{C}_{z z}\left(q_{\|}\right) . q_{z}$ is the wave vector transfer normal to the surface and $\rho$ is the electron density of the liquid; $\sigma$ denotes the surface roughness due to capillary waves. Equation (24) consists of a static term representing the scattering from the mean surface and a fluctuating part stemming from the capillary waves. It has been noted earlier ${ }^{5,22}$ that this form allows for heterodyne mixing, i.e., the intensity autocorrelation function contains besides the usual quadratic terms $\left[\widetilde{C}_{z z}\left(q_{\|}, \tau\right)\right]^{2}$ also terms linear in the correlation functions $\widetilde{C}_{z z}(q, \tau)$. To see this we insert Eq. (24) into Eq. (23) and obtain 


$$
\begin{aligned}
g_{2}\left(q_{\|}, \tau\right)= & +\frac{1}{\left(H\left(q_{\|, 0}\right)+q_{z}^{2} \int d q_{\|}^{\prime} \widetilde{C}_{z z}\left(q_{\|}^{\prime}, 0\right) H\left(q_{\|}, q_{\|}^{\prime}\right)\right)^{2}} \\
& \times\left[F\left(q_{\|}, 0,0\right)+2 q_{z}^{2} \int d q_{\|}^{\prime \prime} F\left(q_{\|}, 0, q_{\|}^{\prime \prime}\right) \widetilde{C}_{z z}\left(q_{\|}^{\prime \prime}, \tau\right)\right. \\
& \left.+q_{z}^{4} \iint d q_{\|}^{\prime} d q_{\|}^{\prime \prime} F\left(q_{\|}, q_{\|}^{\prime}, q_{\|}^{\prime \prime}\right) \widetilde{C}_{z z}\left(q_{\|}^{\prime}, \tau\right) \widetilde{C}_{z z}\left(q_{\|}^{\prime \prime}, \tau\right)\right] .
\end{aligned}
$$

The relative weight of the linear and quadratic terms in the intensity autocorrelation function depends on the resolution parameter $\Delta$, on the magnitude of the diffuse signal $\left\langle C_{z z}\left(q_{\|}\right)\right\rangle$, and on the wave vector transfer $q_{z}$. A large value of $q_{z}$ and a large value of the magnitude of the correlation function $\widetilde{C}\left(q_{\|}\right)$ favor the quadratic term in Eq. (25). The linear term, however, becomes significant for large values of the wave vector spread $\Delta q$.

\section{B. Wave vector spread}

The resolution parameter $\Delta$ represents the wave vector spread or experimental resolution of the setup for a pointlike detector. The calculation of $\Delta$ is based on a Gaussian Schellmodel source and an approximation is given for the $x$ direction (along the beam) ${ }^{19}$

$$
\Delta q_{x}=\frac{1}{\Delta}=2 \pi \sqrt{\frac{2}{\Xi_{x}^{2}}+\frac{1}{\Sigma_{x}^{2}}\left[1+\left(\frac{k_{0} \Delta a^{2}}{L_{a}}\right)^{2}\left(1-\frac{\Sigma_{x}^{2}}{\Delta a^{2}} \frac{L_{a} \sin ^{2}\left(\alpha_{f}\right)+L_{b}^{2} \sin ^{2}\left(\alpha_{i}\right)}{L_{b}}\right)^{2}\right]}
$$

and in the $y$ direction (perpendicular to the beam)

$$
\begin{aligned}
\Delta q_{y} & =\frac{1}{\Delta} \\
& =2 \pi \sqrt{\frac{2}{\Xi_{y}^{2}}+\frac{1}{\Sigma_{y}^{2}}\left[1+\left(\frac{k_{0} \Delta a^{2}}{L_{a}}\right)^{2}\left(1-\frac{\Sigma_{y}^{2}}{\Delta a^{2}} \frac{L_{a}+L_{b}}{L_{b}}\right)^{2}\right]} .
\end{aligned}
$$

$\Delta a$ is a measure for the opening $\Psi$ of the incident aperture via $\Psi_{x, y}=\sqrt{2} \Delta a_{x, y}$. $\Xi_{x, y}$ denotes the respective projected transverse coherence length and $\Sigma_{x, y}$ the projected beam size on the sample. Using typical experimental parameters, we find the wave vector spread to be on the order of $10^{-7} \AA^{-1}$. It depends linearly on the exit angle $\alpha_{f}$, i.e., it increases with increasing exit angle. The out-of-plane direction is not affected by the elongated footprint and therefore the wave vector spread is considerably larger with typical values around $10^{-5} \AA^{-1} . \Delta q_{y}$ does not depend on the exit angle. We note that the ratio $\Delta q / q$ is usually very large (around $10^{-1}$ ) for grazing incidence angles, which results in dramatic effects of partial coherence on the measured correlation functions. For bulk samples the wave vector transfers obtained are usually larger, the ratio $\Delta q / q$ is of the order of $10^{-2}$ or less, and effects of partial coherence are less important. The effects of detector opening, however, are still important, and our main result represents a rigorous approach to calculating the correct XPCS signal.

\section{Surface height-height correlation function}

The height-height correlation function $\widetilde{C}_{z z}\left(q_{\|}, \tau\right)$ can be obtained by Fourier transform of the dynamic structure factor $S\left(q_{\|}, \omega\right)$ of the surface fluctuations. The power spectrum can be calculated with the help of linear response theory from the linearized Navies-Stokes equation with appropriate boundary conditions at the surface and interface. As a starting point, we write the dispersion relation of surface waves as

$$
\begin{aligned}
D(\omega)= & {\left[i \omega \eta\left(q_{\|}+m\right)\right]\left[\gamma q_{\|}^{2}+i \omega \eta\left(q_{\|}+m\right)-\varrho \omega^{2} / q_{\|}\right]-[i \omega \eta(m} \\
& \left.\left.-q_{\|}\right)\right]^{2},
\end{aligned}
$$

where $m=\sqrt{q_{\|}^{2}+i \omega \rho / \eta}, \gamma$ is the surface tension, $\eta$ the viscosity of the bulk phase, and $\varrho$ the mass density of the bulk phase. Solving the equation $D(\omega)=0$ gives an expression for the dispersion relation. The dynamic structure factor of the transverse surface waves is given by

$$
S_{z z}\left(q_{\|}, \omega\right)=\frac{2 k_{B} T}{\pi \omega} \operatorname{Im}\left(\frac{i \omega \eta\left(m+q_{\|}\right)}{D(\omega)}\right) .
$$

Now the time-dependent height-height correlation function $\widetilde{C}_{z z}\left(q_{\|}, \tau\right)=F T\left\{S\left(q_{\|}, \omega\right)\right\}$ of capillary waves can be calculated. If the condition $\left(2 \nu q_{\|}\right) / \omega_{\mathrm{cw}} \ll 1$ is satisfied, where $\nu$ denotes the kinematic viscosity and $\omega_{\mathrm{cw}}$ the capillary wave frequency $\omega_{\mathrm{cw}}^{2}=\gamma q_{\|}^{3} / \varrho$, the height-height correlation function can be approximated as

$$
\widetilde{C}_{z z}\left(q_{\|}, \tau\right)=\frac{k_{B} T}{\gamma q_{\|}^{2}} \cos (\omega \tau) e^{-\Gamma_{1} \tau},
$$

where $\Gamma_{1}$ denotes the damping constant given by $\Gamma_{1}$ $=2 \eta q_{\|}^{2} / \varrho$. For the other limit, i.e., for highly viscous liquids, the condition $\left(2 \nu q_{\|}\right) / \omega_{\mathrm{cw}} \gg 1$ is valid and the correlation function can be approximated as

$$
\widetilde{C}_{z z}\left(q_{\|}, \tau\right)=\frac{k_{B} T}{\gamma q_{\|}^{2}} e^{-\Gamma_{2} \tau},
$$

with $\Gamma_{2}=\frac{\gamma}{2 \eta} q_{\|}$. For a thin liquid film of thickness $h$ the expression Eq. (31) is also valid with a damping constant $\Gamma_{3}$ 


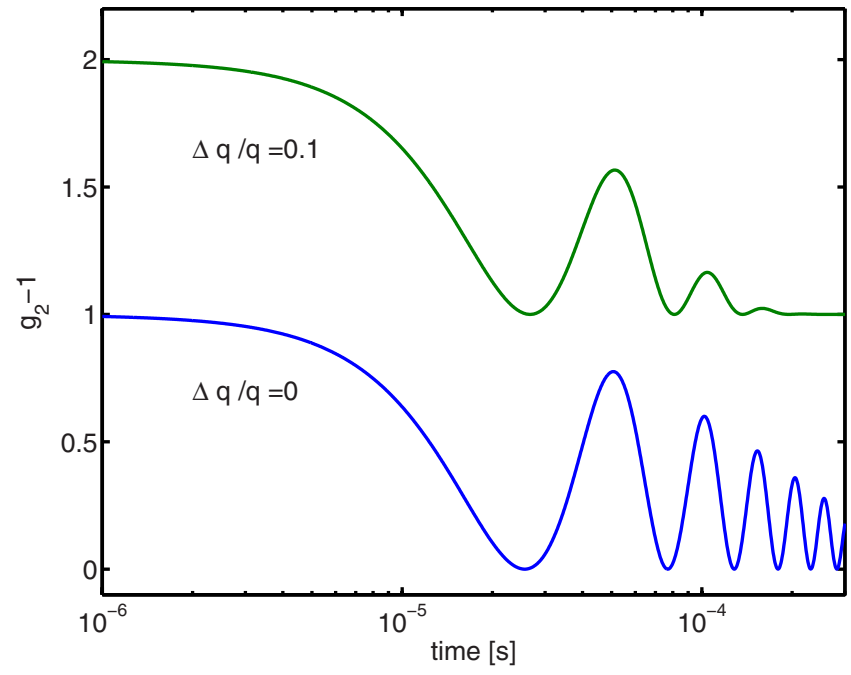

FIG. 2. (Color online) Calculated propagating intensity autocorrelation functions at $q_{\|}=5 \times 10^{-6} \AA^{-1}$. The lower curve represents $g_{2}\left(q_{\|}, \tau\right)$ without any effects of partial coherence, i.e., at infinitely good resolution. The upper curve (shifted +1 for clarity) represents $g_{2}\left(q_{\|}, \tau\right)$ as calculated from Eq. (25) with a relative resolution $\Delta q / q=0.1$. The two functions are calculated for a pointlike detector.

$=\gamma_{\|}[\sinh (q h) \cosh (q h)-q h] /\left[2 \eta \cosh ^{2}(q h)+(q h)^{2}\right] .^{3}$

We will now discuss the properties of the intensity autocorrelation function with regard to these two limiting cases.

\section{EXAMPLES}

Equations (23) and (25) represent the master formulas for calculating any intensity autocorrelation function, taking all kinds of resolution effects into account. In this section we illustrate the importance of resolution effects for selected examples of correlation functions from liquid surfaces.

Figure 2 shows as an illustration the effects of partial coherence on the intensity autocorrelation function from a liquid water surface measured under grazing incidence angles. The correlation function has been calculated using Eq. (30) with the viscosity of water $\eta=10^{-3} \mathrm{~Pa} \mathrm{~s}$, the density $\rho=1000 \mathrm{~kg} / \mathrm{m}$, and the surface tension $\gamma=0.072 \mathrm{~N} / \mathrm{m}$. The wave vector transfer perpendicular to the surface was $q_{z}=0.014 \AA^{-1}$ and the parallel wave vector transfer $q_{\|}=5$ $\times 10^{-6} \AA^{-1}$. In order to separate effects due to partial coherence and finite detector area, we assumed for the first example the case of an infinitely small detector area, i.e., $\beta$ $=0$ in Eqs. (19) and (22). This leads automatically to a contrast $g_{2}(q, 0)-1=1$. The bottom line in Fig. 2 represents the correlation function $g_{2}(q, \tau)-1$ without taking any resolution effects into account, i.e., $\Delta q=0$ in Eqs. (19) and (22). The intensity autocorrelation function is then identical to the absolute square of the height-height correlation function of the surface, and a refinement of the data would yield immediately the values of surface tension and viscosity. In contrast, the upper curve in Fig. 2 displays an intensity correlation function including effects of partial coherence. The relative wave vector spread is $\Delta q / q=0.1$. Apparently, the effect of partial coherence does not alter the propagation frequency of the correlation function but does alter the damping constant,

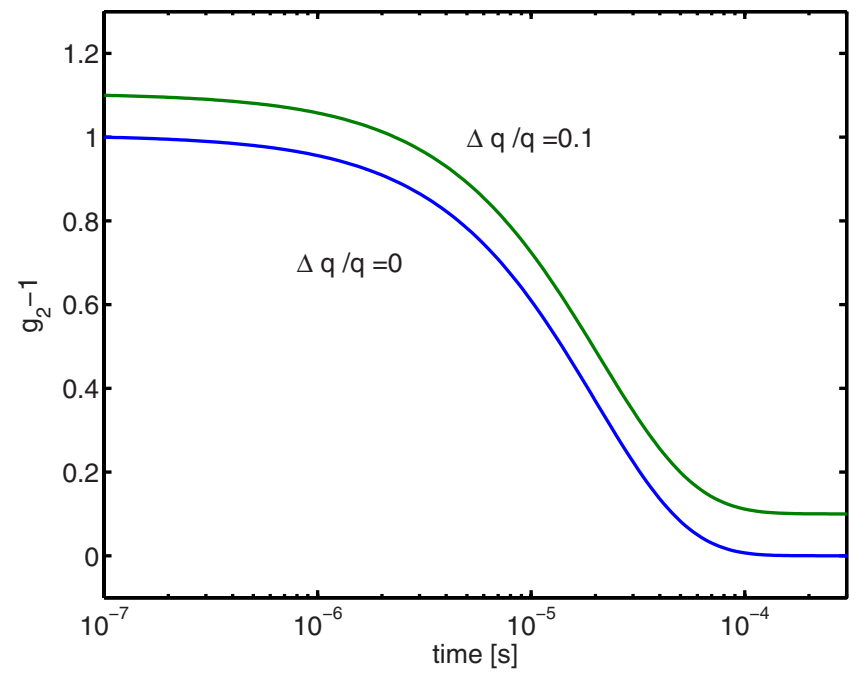

FIG. 3. (Color online) Calculated overdamped intensity autocorrelation functions at $q_{\|}=5 \times 10^{-6} \AA^{-1}$. The lower curve represents $g_{2}\left(q_{\|}, \tau\right)$ without any effects of partial coherence, i.e., at infinitely good resolution. The upper curve (shifted +1 for clarity) represents $g_{2}\left(q_{\|}, \tau\right)$ as calculated from Eq. (25) with a relative resolution $\Delta q / q=0.1$. The two functions are calculated for a pointlike point detector.

which appears to be considerably higher than the bulk value. This problem is particularly severe for propagating waves and was first addressed by Langevin et al. ${ }^{16}$ It can be easily understood in frequency space, where propagating waves are represented by Lorentzians centered at the propagation frequency $\omega_{c}$ with a linewidth $\Gamma_{1}$. A finite resolution leads to a superposition of Lorentzians slightly shifted in the center frequency. The resulting Lorentzian is still centered at the mean frequency $\omega_{c}$ but provides a considerably larger linewidth due to the superposition. The resulting damping constant appears to be much higher than is given by the viscosity of the material.

Figure 3 displays essentially the same situation as in Fig. 2 , with the exception of a higher viscosity. The viscosity is $\eta=0.05 \mathrm{~Pa} \mathrm{~s}$, yielding overdamped capillary waves on the surface. Again the bottom line shows the correlation function for zero wave vector spread and the top line the function with a spread. The influence of the resolution function on the intensity autocorrelation function is less dramatic than for propagating waves. In fact, under the presented conditions the relaxation times of the two intensity correlation functions are almost identical. This can be understood again by switching to frequency space. The overdamped waves on highviscosity materials are Lorentzian lines centered at zero frequency, i.e., this is a quasielastic component. This is true for all waves within the resolution volume, which all appear at zero frequency transfer. Accordingly, the smearing out effect is much less dramatic than for propagating waves.

This, however, is not the only influence of partial coherence. An even stronger influence of the resolution on the functional form of the intensity autocorrelation function can be observed when the resolution is decreased, i.e., the ratio $\Delta q / q$ is increased. Figure 4 shows correlation functions for different values of $\Delta q / q$ ranging from 0.197 to 0.210 (from 


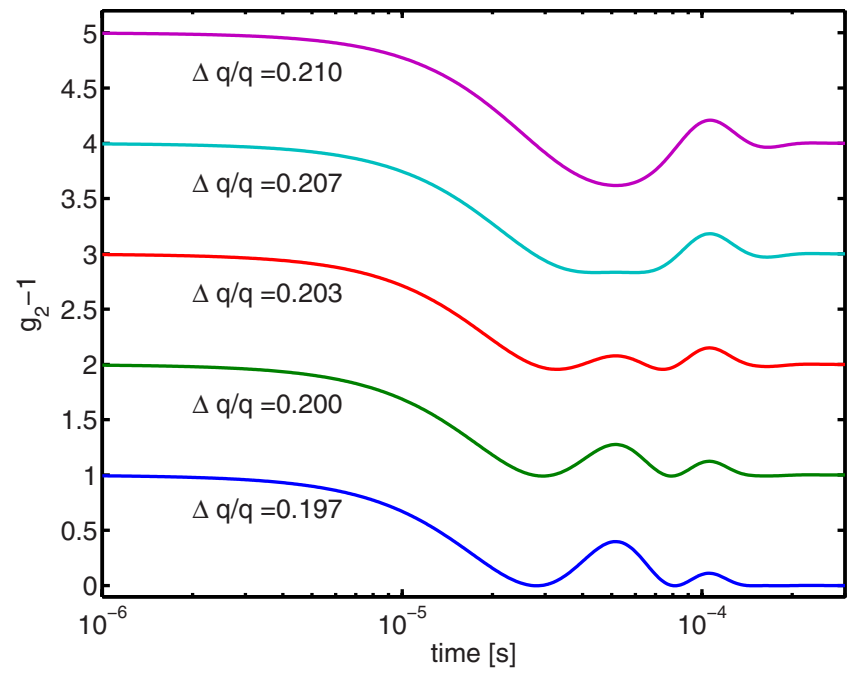

FIG. 4. (Color online) Calculated propagating intensity autocorrelation functions at $q_{\|}=5 \times 10^{-6} \AA^{-1}$ for different values of the wave vector spread (from bottom to top, $\Delta q / q=0.197,0.2,0.203$, 0.207 , and 0.21 ). The correlation functions are shifted for reasons of clarity. All functions are calculated for a pointlike detector.

bottom to top). Again the calculation has been performed assuming a pointlike detector $(\beta=0)$. For reasons of clarity and visibility, all correlation functions have been normalized to their maximum value at $\tau=0$ and shifted. The two correlation functions with $\Delta q / q=0.197$ and 0.2 display the usual propagating capillary wave correlation function proportional to $\cos ^{2}(\omega \tau)$ with a beating above the baseline. This form changes when the wave vector spread increases to $\Delta q / q$ $=0.203$; now the amplitude of the first oscillation is lower than that of the second. At even higher values of $\Delta q / q$, the character of the intensity autocorrelation function changes entirely from a $g(q, \tau) \sim \cos ^{2}(\omega \tau)$ behavior to a $g(q, \tau)$ $\sim \cos (\omega \tau)$ behavior, i.e., from an oscillation above a baseline to an oscillation around the baseline with a decrease in frequency. Thus, we observe a transition from a homodyne to a heterodyne correlation function as a function of wave vector spread $\Delta q / q$. The correlation function at larger values of the wave vector spread is no longer proportional to the absolute square of the height-height correlation function but to the real part of it. The heterodyne correlation function is due to a mixing between the dynamic signal stemming from the surface fluctuations and the static reference signal originating from the mean surface. This has been observed experimentally for the case of capillary waves on liquid water surfaces and can be explained by assuming a wave vector spread of the order of $\Delta q \approx 1 \times 10^{-7} \AA^{-1}$. This intrinsic mixing has also been observed in the case of fluctuations of smectic membranes. Our calculation represents a rigorous explanation of these observations. It is also evident that the excessive damping measured in Ref. 5 is due to resolution effects.

While heterodyning always leads to a decrease of contrast, the optical mixing may be advantageous from another point of view. Under certain conditions the signal to noise ratio of the correlation function can be increased and heterodyne mixing of signals relaxes the condition of using the Siegert relation, which is restricted to Gaussian statistics. In

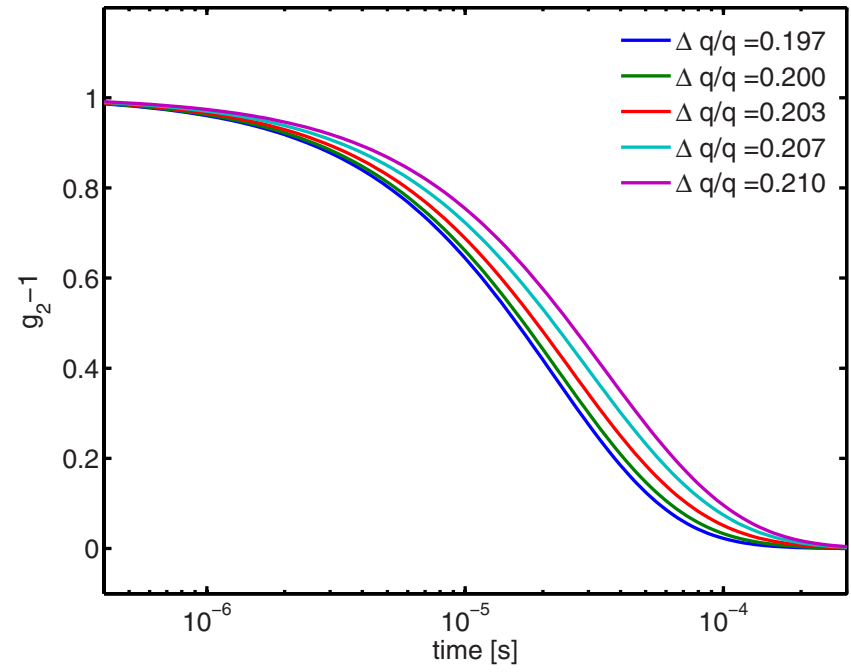

FIG. 5. (Color online) Calculated overdamped intensity autocorrelation functions at $q_{\|}=5 \times 10^{-6} \AA^{-1}$ for different values of the wave vector spread (from left to right, $\Delta q / q=0.197,0.2,0.203$, 0.207 , and 0.21 ). All functions are calculated for $\beta=0$, i.e., for a pointlike detector.

this context, we may note that external heterodyne mixing using a static reference signal from outside the fluctuating sample has been achieved recently. ${ }^{23}$

It is clear that a similar transition from homodyne to heterodyne correlation functions can be achieved by decreasing the overall strength of the fluctuating signal. This implies that we expect, for example, that liquids providing a low surface tension would predominantly show homodyne, i.e., $g(q, \tau) \sim \cos ^{2}(\omega \tau)$, correlation functions, while high-surfacetension materials like, e.g., liquid metals, would rather show heterodyne correlation functions for the same wave vector spread.

Figure 5 shows the same homodyne to heterodyne transition for the case of overdamped capillary waves as present, e.g., on polymer films. Here the transition is less evident than in the previous case, as the oscillating term in the correlation function is missing. Nevertheless, we also observe a decrease of the relaxation time with increasing wave vector spread. That is, the correlation function at $\Delta q / q=0.21$ is a heterodyne correlation function with $g(q, \tau) \approx \exp \left(-\Gamma_{2} \tau\right)$, in contrast to the homodyne correlation function $g_{2}(q, \tau)$ $\approx\left[\exp \left(-\Gamma_{2} \tau\right)\right]^{2}$. In the transition region, we observe the sum of both exponential functions, leading to the impression that the correlation function shows a stretched exponential behavior. This demonstrates again the importance of incorporating the resolution effects into the modeling of XPCS data.

Up to now, we have calculated the correlation functions assuming an infinitely good detector resolution. This condition will be relaxed in order to demonstrate the effects of finite detector opening on the correlation functions. Figure 6 displays intensity autocorrelation functions corresponding to propagating capillary waves on a water surface with a wave vector spread $\Delta q / q=0.06$ for detector openings ranging from 0.3 to $120 \mu \mathrm{m}$. Under these conditions, the correlation function is of homodyne character and the influence of the detector opening is primarily visible as a decrease of the contrast 


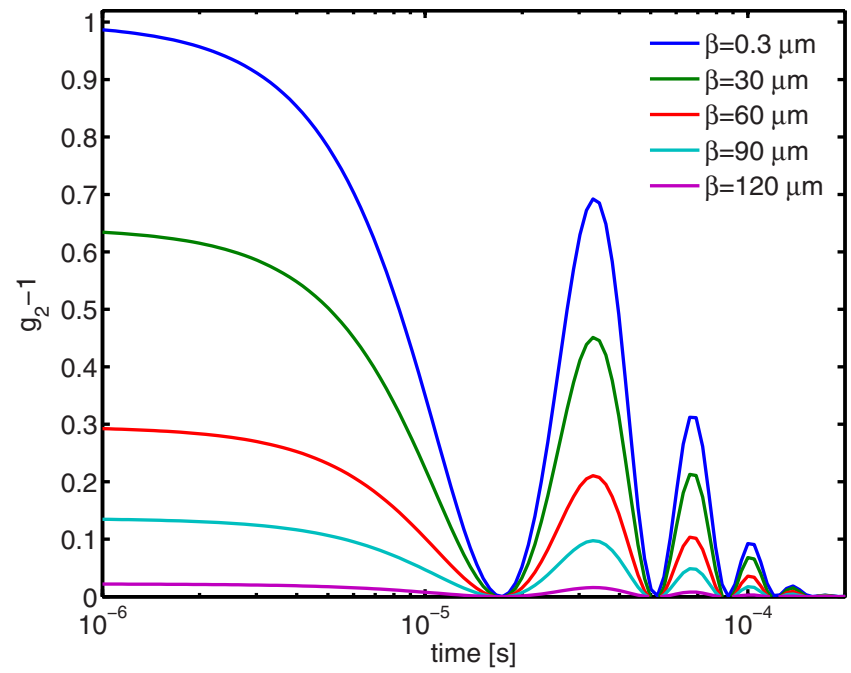

FIG. 6. (Color online) Calculated propagating intensity autocorrelation functions at $q_{\|}=5 \times 10^{-6} \AA^{-1}$ for different values of the detector opening (from top to bottom, $\beta=0.3,30,60,90$, and $120 \mu \mathrm{m})$. All functions are calculated for fixed value of the wave vector spread $\Delta q / q=0.06$.

with increasing detector area. With this particular small value of $\Delta q / q=0.06$, the frequencies and the damping constants are not affected by the detector resolution.

Figure 7 shows overdamped correlation functions with a wave vector spread $\Delta q / q=0.06$ for detector openings ranging from 0.3 to $120 \mu \mathrm{m}$. Again all correlation functions have been normalized to unity. As previously, the correlation functions are only slightly affected by the increasing detector opening.

At this value of the wave vector spread the main effect of the detector resolution is to decrease the contrast as a function of detector opening. The contrast $c$ is defined as the

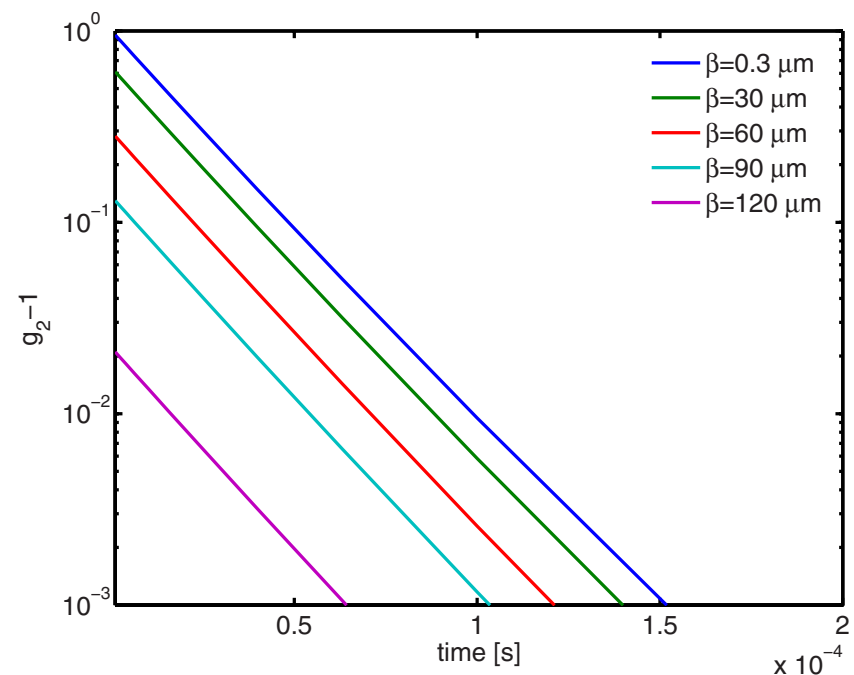

FIG. 7. (Color online) Calculated overdamped intensity autocorrelation functions at $q_{\|}=5 \times 10^{-6} \AA^{-1}$ for different values of the detector opening (from top to bottom, $\beta=0.3,30,60$, 90, and $120 \mu \mathrm{m})$. All functions are calculated for fixed value of the wave vector spread $\Delta q / q=0.06$. Note that the correlation function is now plotted on a logarithmic scale with the time axis being linear.

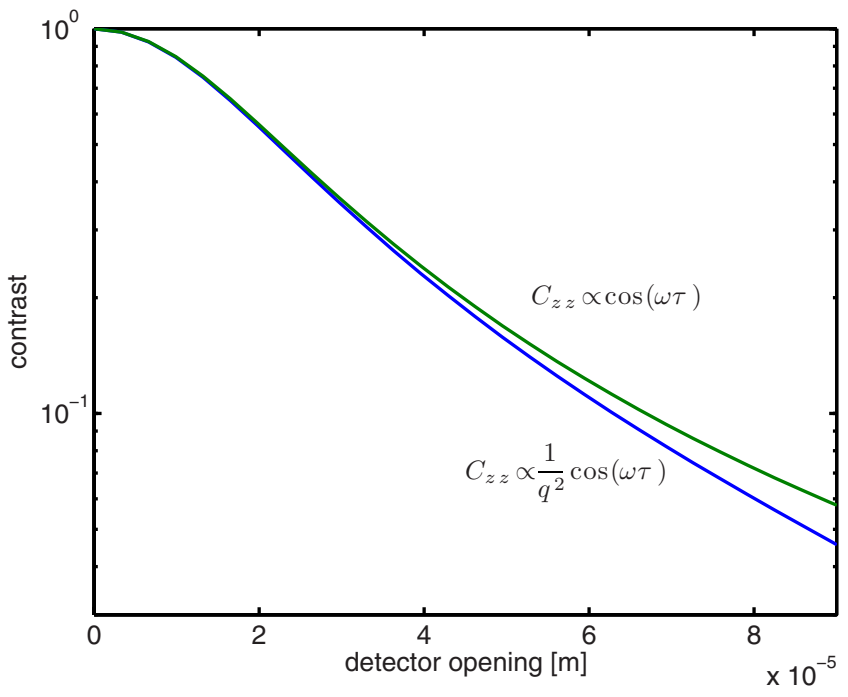

FIG. 8. (Color online) Contrast $\left[g_{2}\left(q_{\|}, 0\right)-1\right]$ as a function of detector opening of two surface correlation functions with different $q$ dependence. The upper curve represents the contrast for $C_{z z}$ $=\cos (\omega \tau)$ while the lower curve is calculated with $C_{z z}$ $=q_{\|}^{-2} \cos (\omega \tau)$. Calculated at $q_{\|}=5 \times 10^{-6} \AA^{-1}$ with a fixed wave vector spread $\Delta q / q=0.06$.

intercept of the correlation function at zero lag time, $c$ $=g_{2}(0)-1$. From an inspection of the expression Eq. (23), it is evident that the contrast is in general not an independent quantity which can be separated from the properties of the sample. In order to illustrate this, we computed the contrast as a function of detector opening for two correlation functions which differ in their $q$ dependence. The upper curve in Fig. 8 represents the contrast for $\widetilde{C}_{z z}=\cos (\omega \tau)$, while the lower curve belongs to a correlation function $\tilde{C}_{z z}$ $=q_{\|}^{-2} \cos (\omega \tau)$ showing the capillary-wave-like $q^{-2}$ behavior. It can be seen that already at a detector opening of $60 \mu \mathrm{m}$ the two curves differ considerably. Therefore, it is in general difficult to deduce reliably the coherence properties of an $\mathrm{x}$-ray beam by measuring the contrast of intensity correlations and neglecting the sample properties.

The detector opening may also affect the form of the intensity autocorrelation function and can also induce a transition from a homodyne to a heterodyne correlation function similar to the one observed by altering the wave vector spread or changing the magnitude of the fluctuating signal. Figure 9 displays correlation functions of a liquid water surface at detector openings ranging from 0.3 to $120 \mu \mathrm{m}$. All correlation functions are normalized by their maximum value. The wave vector spread is $\Delta q / q=0.206$. At small detector openings, the correlation function is of heterodyne character, shown by the $\cos (\omega \tau)$ behavior. Increasing the detector opening results in a shift to smaller time scales. This is due to an increasing fraction of the homodyne correlation function proportional to $\cos ^{2}(\omega \tau)$, which is becoming visible at a detector opening of $60 \mu \mathrm{m}$. At $120 \mu \mathrm{m}$ the correlation function is again completely dominated by the homodyne function.

Figure 10 shows the same case as in Fig. 9 but for overdamped correlation functions. In order to visualize the influ- 


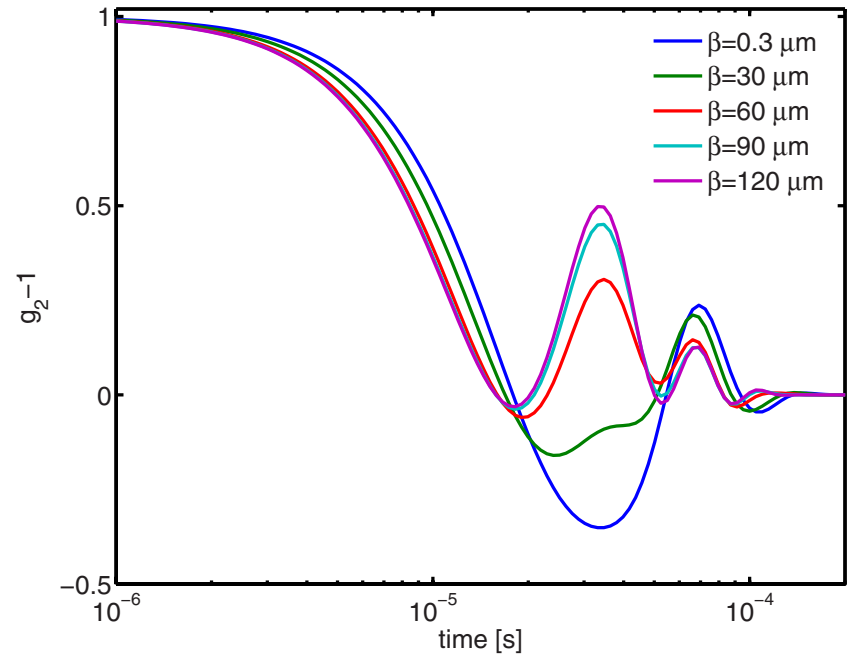

FIG. 9. (Color online) Calculated propagating intensity autocorrelation functions at $q_{\|}=5 \times 10^{-6} \AA^{-1}$ and fixed wave vector spread $\Delta q / q=0.206$ for different values of the detector opening $\beta$.

ence on the shape of $g_{2}$, we normalized all correlation functions to unity. Again the transition from homodyne to heterodyne correlation function is visible as the detector opening is increased. In the transition region the correlation function shows a strong stretching behavior.

\section{SUMMARY AND CONCLUSION}

We have presented a rigorous formalism for the effects of partial coherence and detector resolution on XPCS intensity autocorrelation functions. Starting from first principles using Huygens-Fresnel propagation theory and the first Born ap-

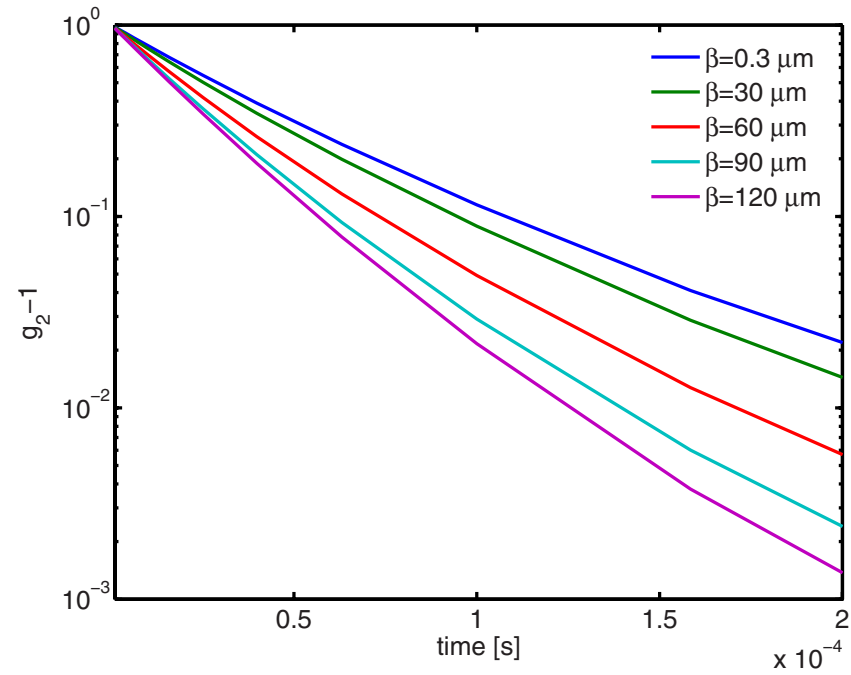

FIG. 10. (Color online) Calculated overdamped intensity autocorrelation functions at $q_{\|}=5 \times 10^{-6} \AA^{-1}$ and fixed wave vector spread $\Delta q / q=0.206$ for different values of the detector opening.

proximation, we derived under the assumption of spatial homogeneity of the electron density correlation function a general expression resembling the classical resolution folding approach. It is shown that the effects of partial coherence and detector resolution can be cast into two general resolution functions which enter the normalized intensity autocorrelation function. The results are illustrated by application to the case of scattering from liquid surfaces. The examples demonstrate that XPCS experiments from surfaces must be interpreted with caution, as the resulting intensity autocorrelation functions are strongly affected by detector resolution and partial coherence.
${ }^{1}$ T. Seydel, A. Madsen, M. Tolan, G. Grübel, and W. Press, Phys. Rev. B 63, 073409 (2001).

${ }^{2}$ I. Sikharulidze, I. P. Dolbnya, A. Fera, A. Madsen, B. I. Ostrovskii, and W. H. de Jeu, Phys. Rev. Lett. 88, 115503 (2002).

${ }^{3}$ H. Kim, A. Rühm, L. B. Lurio, J. K. Basu, J. Lal, D. Lumma, S. G. J. Mochrie, and S. K. Sinha, Phys. Rev. Lett. 90, 068302 (2003).

${ }^{4}$ A. Madsen, J. Als-Nielsen, and G. Grübel, Phys. Rev. Lett. 90, 085701 (2003).

${ }^{5}$ C. Gutt, T. Ghaderi, V. Chamard, A. Madsen, T. Seydel, M. Tolan, M. Sprung, G. Grübel, and S. K. Sinha, Phys. Rev. Lett. 91, 076104 (2003).

${ }^{6}$ A. Madsen, T. Seydel, M. Sprung, C. Gutt, M. Tolan, and G. Grübel, Phys. Rev. Lett. 92, 096104 (2004).

${ }^{7}$ X. Hu, Z. Jiang, S. Narayanan, X. Jiao, A. Sandy, S.K. Sinha, L.B. Lurio, and J. Lal, Phys. Rev. E 74, 010602(R) (2006).

${ }^{8}$ Z. Jiang, H. Kim, X. Jiao, H. Lee, Y.-J. Lee, Y. Byun, S. Song, D. Eom, C. Li, M. H. Rafailovich, L. B. Lurio, and S. K. Sinha, Phys. Rev. Lett. 98, 227801 (2007).

${ }^{9}$ S. Streit, C. Gutt, V. Chamard, M. Sprung, A. Robert, H. Sternemann, and M. Tolan, Phys. Rev. Lett. 98, 047801 (2007).
${ }^{10}$ S. Narayanan, D. R. Lee, A. Hagman, X. Li, and J. Wang, Phys. Rev. Lett. 98, 185506 (2007).

${ }^{11}$ C. Gutt, M. Sprung, R. Fendt, A. Madsen, S. K. Sinha, and M. Tolan, Phys. Rev. Lett. 99, 096104 (2007).

${ }^{12}$ M. Sutton, S. G. J. Mochrie, T. Greytag, S. E. Nagler, L. E. Berman, G. A. Held, and G. B. Stephenson, Nature (London) 352, 608 (1991).

${ }^{13}$ J. L. Libbert, R. Pindak, S. B. Dierker, and I. K. Robinson, Phys. Rev. B 56, 6454 (1997).

${ }^{14}$ Light Scattering by Liquid Surfaces and Complementary Techniques, edited by D. Langevin (Marcel Dekker, New York, 1992).

${ }^{15}$ J. C. Earnshaw, Appl. Opt. 36, 7583 (1997).

${ }^{16}$ D. Langevin, J. Chem. Soc., Faraday Trans. 1 70, 95 (1974).

${ }^{17}$ I. Sikharulidze, I. P. Dolbnya, A. Fera, A. Madsen, and W. H. de Jeu, Opt. Commun. 247, 111 (2005).

${ }^{18}$ S. K. Sinha, M. Tolan, and A. Gibaud, Phys. Rev. B 57, 2740 (1998).

${ }^{19}$ T. Ghaderi, Ph.D. thesis, University of Dortmund, Germany, 2006, http://eldorado.uni-dortmund.de:8080/handle/2003/22293

${ }^{20}$ P. Pusey, in Photon Correlation Spectroscopy and Velocimetry, 
edited by H. Z. Cummins and E. R. Pike (Plenum Press, New York, 1977), p. 236.

${ }^{21}$ M. Tolan and S. K. Sinha, Physica B 48, 399 (1998).

${ }^{22}$ I. Sikharulidze, B. Farago, I. P. Dolbnya, A. Madsen, and W. H. de Jeu, Phys. Rev. Lett. 91, 165504 (2003).

${ }^{23}$ F. Livet, F. Bley, F. Ehrburger-Dolle, I. Morfin, E. Geissler and M. Sutton, J. Synchrotron Radiat. 13, 453 (2006). 